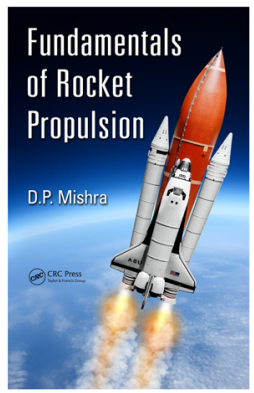

\section{Fundamentals of Rocket Propulsion}

\section{P. Mishra}

CRC Press, Taylor \& Francis Group, 6000 Broken Sound Parkway NW, Suite 300, Boca Raton, FL, 33487-2742, USA. 2017. Distributed by Taylor \& Francis Group, 2 Park Square, Milton Park, Abingdon, OX14 4RN, UK. 461pp. Illustrated. £89. (20\% discount available to RAeS members via www. crcpress.com using AKQ07 promotion code). ISBN 978-1-4987-8535-8.

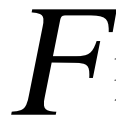
Tundamentals of rocket propulsion provides a comprehensive introduction to rocket propulsion systems for space applications.

The main focus of the book is thermochemical rocket propulsion systems and the introductory chapters include a useful review of key aerothermodynamic principles. Subsequent chapters cover key performance parameters for thermochemical systems and details of nozzle flow as well as a chapter covering spacecraft flight analysis, including atmospheric launch vehicle analysis and basic orbit mechanics relating to propulsion.
A chapter on the types of propellants for thermochemical systems specifically for solid, liquid and hybrid propulsion systems provides a good general introduction to propellants and their properties. This chapter is then followed by specific chapters individually covering the basic operation and performance analysis of solid, liquid and hybrid systems. One minor omission here seems to be cold gas propulsion systems which are not treated specifically in the text.

In addition to the more comprehensive treatment of thermochemical propulsion systems, there is a short introductory chapter on non-chemical rocket propulsion systems which in the main focuses on introducing the basic principles of electric propulsion systems. This includes some basic energy based models for electro-thermal and electro-static systems alongside a description of the principles of operation of electro-magnetic thrusters including some key performance and scaling parameters. In addition, there is a short description of the basic principles of operation of nuclear and solar energy rocket motors.

Throughout the chapters where analytical methods are introduced example problems with worked examples are included which help the reader understand the application of the analysis methods for basic design estimations. Each chapter of the book finishes with some relevant review questions which serve as revision guide and a test of understanding for anyone working through the material. Additionally, some chapters also include numerical problems which provide an additional resource to test understanding of the various analysis methods. However, it would have been nice to have seen the 
inclusion of the numerical solutions to these problems.

Overall, this is a very good undergraduate level text book covering basics of thermochemical rocket propulsion elements and introducing key principles of other rocket propulsion systems.

Dr Katharine Smith, MRAeS

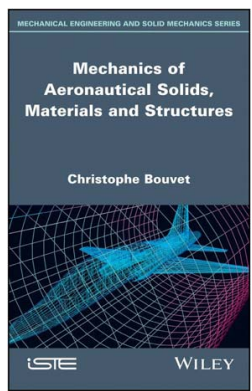

\section{Mechanics of Aeronautical Solids, Materials and Structures}

\section{Bouvet}

ISTE Ltd. and John Wiley and Sons, The Atrium, Southern Gate, Chichester, West Sussex, PO19 8SQ. 2017. xiv; 284pp. Illustrated. £100. ISBN 978-1-78630-115-4.

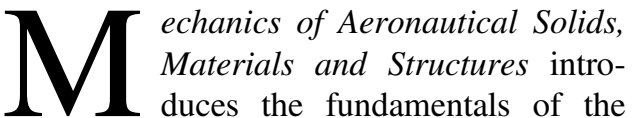
mechanics of materials to audiences interested in airframe structures. The book is aimed at presenting the key elements and concepts that any structural engineer needs to be aware off, the fundamentals of stress, strain and their constitutive relations; the experimental behaviour of materials along with the material properties and applicable failure theories; the methods and the design tools for analysing airframe structures. The book is mainly targeted for undergraduate aerospace engineering students.

The book opens with the definition and explanation of fundamental concepts such as stress, strain and their constitutive relation. The concepts are presented adequately, but 